\title{
Sodium selenite inhibits $\gamma$-secretase activity through activation of ERK
}

\author{
Ying-Tsen Tunga,b, Wen-Ming $\mathrm{Hsu}^{\mathrm{c}}$, Bo-Jeng Wang ${ }^{\mathrm{a}}$, Shin-Ying Wu ${ }^{\mathrm{a}}$, Chen-Tung Yen ${ }^{\mathrm{b}}$, \\ Ming-Kuan $\mathrm{Hu}^{\mathrm{d}, *}$, Yung-Feng Liao ${ }^{\mathrm{a}, \mathrm{b}, * *}$ \\ a Laboratory of Molecular Neurobiology, Institute of Cellular and Organismic Biology, Academia Sinica, Taipei, Taiwan \\ ${ }^{\mathrm{b}}$ Institute of Zoology, National Taiwan University, Taipei, Taiwan \\ c Department of Surgery, National Taiwan University Hospital, National Taiwan University College of Medicine, Taipei, Taiwan \\ d School of Pharmacy, National Defense Medical Center, 161, Sec. 6, Min-Chuan E. Road, Taipei 114, Taiwan
}

\section{A R T I C L E I N F O}

\section{Article history:}

Received 26 February 2008

Received in revised form 14 May 2008

Accepted 14 May 2008

\section{Keywords:}

Alzheimer's disease

Amyloid- $\beta$

MEK1

Nicastrin

Presenilin-1

C99

Phosphorylation

\begin{abstract}
A B S T R A C T
Previous studies have demonstrated that the ERK MAPK acts as a negative regulator of $\gamma$-secretase. Here, we demonstrate that the activation of ERK MAPK pathway by sodium selenite can inhibit endogenous $\gamma$ secretase activity. Consistently, the $\gamma$-secretase-mediated production of amyloid- $\beta(A \beta)$ was dramatically attenuated by sodium selenite in a temporal manner. To substantiate the functional role of ERK MAPK in the regulation of $\gamma$-secretase, we demonstrate that cells transfected with the wild-type MEK1 and a constitutively active mutant of MEK1 also displayed a significant attenuation of $\gamma$-secretase activity. The active purified ERK1/2 can significantly reduce the $\gamma$-secretase-mediated processing of C99, possibly through inducing alterations in the phosphorylation of both nicastrin and presenilin-1. Together, our data suggest that the selenite-elicited ERK activation could effectively reduce $A \beta$ production, supporting that selenium compounds could represent a novel class of nutrient supplements to slow down the progression of Alzheimer's disease.
\end{abstract}

(c) 2008 Elsevier Ireland Ltd. All rights reserved.
Secretase-mediated generation of the amyloid- $\beta(A \beta)$ peptide that is deposited at neuritic plaques has been closely correlated with the pathogenesis of Alzheimer's disease (AD) [17]. The proteolysis of the amyloid precursor protein (APP) by the sequential actions of $\beta$ - and $\gamma$-secretases results in the production of $A \beta$ [2]. Accumulating evidence has demonstrated that presenilin (PS) is the obligatory component of $\gamma$-secretase and binds three additional cofactors, including nicastrin, Aph-1, and Pen-2, to form a multimeric protease complex [7]. The pivotal role of $\gamma$-secretase in the $A \beta$-centered pathogenesis of $A D$ is exemplified by the findings that most FAD-causing mutations are localized to two PS genes and correlate with the selective elevation of $A \beta 42$ versus $A \beta 40[5,18]$. It is thus the $\gamma$-secretase that contributes substantially to the development of $\mathrm{AD}$, and a stringent regulation of $\gamma$-secretase activity is required to maintain the homeostasis of $A \beta$ production.

The molecular mechanism underlying the regulation of the secretase-mediated proteolysis of the APP is just beginning to

\footnotetext{
* Corresponding author. Tel.: +886 2 87923100x18896; fax: +886 287923169 .

** Corresponding author at: Institute of Cellular and Organismic Biology, Academia Sinica, 128 Academia Road, Sec. 2, Taipei 115, Taiwan. Tel.: +886 2 27899535; fax: +886227858059.

E-mail addresses: hmk@ndmctsgh.edu.tw (M.-K. Hu), yliao@sinica.edu.tw (Y.-F. Liao).
}

emerge $[3,20]$. Our previous data clearly reveal that c-Jun $\mathrm{N}$ terminal kinase (JNK) mediates cytokine-elicited stimulation of $\gamma$-secretase activity and $A \beta$ production [11]. All three major MAPK pathways, JNK, extracellular signaling-regulated kinase (ERK), and p38 kinase, are all activated in susceptible regions in the $A D$ brain [23]. Consistently, upstream modulators of the ERK pathway, including Ras, Raf, and MEK1/2, are also activated in AD [12,24,25]. An inorganic selenium compound, sodium selenite, has recently been shown to induce the phosphorylation of p53 and p38 to activate caspase-independent cell death $[10,14]$. Sodium selenite could also significantly activate anti-apoptotic PI3K/AKT and ERK pathways to protect ischemic brain and prostate cancer cells $[6,19]$. These studies thus suggest that selenite could differentially modulate protein phosphorylation to regulate various cellular functions. Given their differential responses to extracellular stimuli, a comprehensive understanding of correlations between these MAPK pathways and the formation of pathological hallmarks in the $A D$ brain could greatly help elucidate the precise mechanism underlying the pathogenesis of AD. Herein, we present evidence for the first time that sodium selenite can activate ERK MAPK pathway to attenuate $\gamma$-secretase-mediated proteolysis.

The BCA protein assay reagent kit and SuperSignal West Dura reagents were purchased from Pierce. Rabbit, anti-ERK1/2 MAP kinase antibody, anti-phospho-ERK1/2 antibody and antiMEK1 antibody were from Cell Signaling Technology. Horseradish 
peroxidase-conjugated anti-rabbit IgG was from Santa Cruz Biotechnology. Horseradish peroxidase-conjugated anti-mouse IgG and ECL Western Blotting detection reagents were from Amersham Biosciences. Dual luciferase assay reagents, Steady-Glo luciferase assay reagents, and pRL-TK vector were from Promega. Lipofectamine 2000 transfection reagent and Dulbecco's modified Eagle's medium (DMEM) were from Invitrogen. Fetal bovine serum (FBS) was from Biological Industries Ltd. (Kibbutz Beit Haemek, Israel). The human $A \beta 40$ colorimetric ELISA kit was from BioSource International. All other reagents were at least reagent grade and were obtained from standard suppliers.

Human embryonic kidney cells (HEK293) were maintained in DMEM supplemented with 10\% FBS. T-REx293 cells were purchased from Invitrogen and cultured in DMEM supplemented with $10 \%$ FBS and $5 \mu \mathrm{g} / \mathrm{ml}$ blasticidin. The generation of C99-GL-T20 (T20) cells and a CHO-derived stable line $\gamma$-30 was described previously [9,11]. $\gamma \mathrm{NCT}-36$ was a $\gamma-30$ derivative that constitutively overexpresses His-tagged nicastrin (NCT), presenilin-1 (PS1), HA-tagged Aph-1, and Flag-tagged Pen-2. Cells were incubated in a humidified incubator at $37^{\circ} \mathrm{C}$ in $5 \% \mathrm{CO}_{2}$.

The quantitative measurement of $\gamma$-secretase activity using T20 cells was previously described [11]. Briefly, cells were treated with various concentrations of sodium selenite in culture medium containing $1 \mu \mathrm{g} / \mathrm{ml}$ tetracycline and incubated at $37^{\circ} \mathrm{C}$ for various intervals. Cells incubated with culture medium containing $1 \mu \mathrm{g} / \mathrm{ml}$ of tetracycline were used to define the basal level of $\gamma$-secretase activity, while cells treated with culture medium without tetracycline were used to estimate the nonspecific background emission of the luciferase signal. The normalized luciferase signal emitted by T20 cells in culture medium without tetracycline was referred to as onefold of activation.

To determine $A \beta$ production, conditioned media from compound-treated or transfected cells were harvested, clarified by centrifugation, supplemented with the Complete ${ }^{\circledR}$ protease inhibitor cocktail, and stored at $-80^{\circ} \mathrm{C}$ until being assayed. Levels of secreted $A \beta 40$ in the conditioned media were determined using a quantitative human $A \beta 40$ sandwich ELISA kit as described in the manufacturer's instructions. A $\beta 40$ measurements were conducted in triplicate, and the medium of T20 cells treated with serum-free DMEM alone was included as the blank.

The MEK1 expressing vector (pCMV-MEK1) encoding the catalytic kinase domain of wild-type mouse MEK1 was purchased from Clontech. To generate the constitutively active (MEK1-EE) and dominant-negative mutants (MEK1-AA) of MEK1 [8], two essential serine residues within its catalytic domain, $\operatorname{Ser}^{218}$ and $\mathrm{Ser}^{222}$, were replaced with glutamic acid (for MEK1-EE) and alanine (for MEK1-AA), respectively, by using the QuickChange site-directed mutagenesis kit according to the manufacturer's instructions.

The detailed procedures of SDS-PAGE and Western blotting were described previously [11]. The immunoblots were visualized by chemiluminescence using the SuperSignal West Dura reagent. Images were captured by and processed with ChemiGenius2 (Syngene). The antibodies used and their dilutions were as follows: anti-Gal4 (Santa Cruz), 1:1000; anti-MEK1 and anti-ERK1/2 (Cell Signaling Technology), 1:1000; HRP-conjugated anti-mouse IgG (Amersham Biosciences) 1:10,000; HRP-conjugated anti-rabbit IgG (Santa Cruz), 1:1000.

Quantitative analysis of Western blots was conducted with the TotalLab v2.01 program by determining the relative density of the immunoreactive bands after acquisition of the blot image with ChemiGenius2 (Syngene). Phosphorylation levels were defined as the ratio of phospho-protein to total protein. Results were expressed as the mean ( \pm S.D.) of triplicate measurements from three independent experiments. Statistical analyses were done by a two-tailed Student's $t$-test. A value of $p<0.05$ was considered significant.

To ascertain whether ERK activation could be an alternative target for the development of novel $A \beta$-lowering agents, we examined the efficacy of a known ERK agonist, sodium selenite, on the inhibition of $\gamma$-secretase and $A \beta$ production. The proteolytic activity of $\gamma$-secretase was determined in cells whose intracellular ERKs were

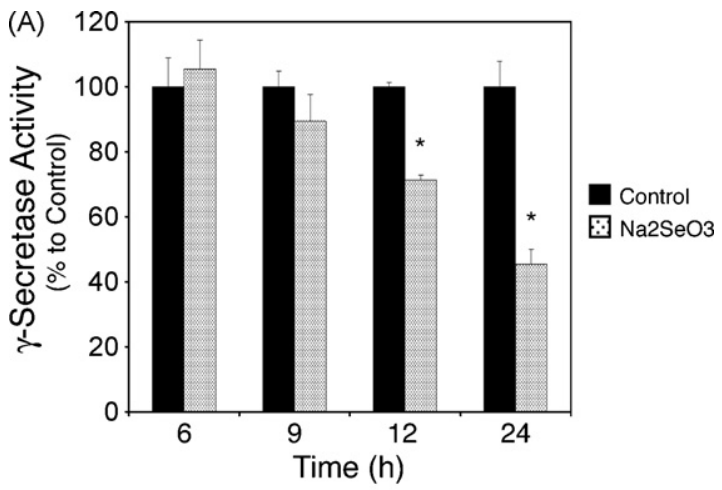

(B)
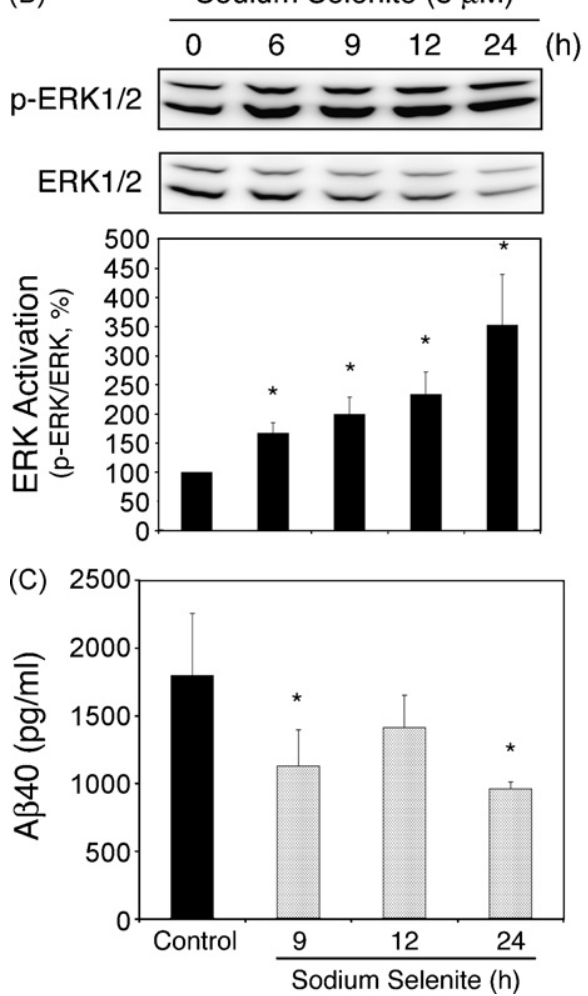

Fig. 1. Sodium selenite antagonizes $\gamma$-secretase activity through activation of ERK. (A) T20 cells were incubated at $37^{\circ} \mathrm{C}$ in the presence or absence of $\mathrm{Na}_{2} \mathrm{SeO}_{3}(8 \mu \mathrm{M})$ for various intervals. $\gamma$-Secretase activity in treated cells (shaded bar) was determined and compared with that of untreated cells (control, solid bar) that is referred to as $100 \%$ relative $\gamma$-secretase activity at each time point. Data are shown as the mean ( \pm S.D.) of triplicate measurements from one representative experiments; ${ }^{*} p<0.05$. (B) A stable CHO cell line $(\gamma-30)$ expressing wild-type human PS1, Aph$1 \alpha 2$, and Pen- 2 was treated with $8 \mu \mathrm{M}$ of $\mathrm{Na}_{2} \mathrm{SeO}_{3}$ and harvested at various intervals. The relative levels of ERK activation (p-ERK1/2 versus total ERK1/2) in treated cells were determined and compared with those of untreated cells that are referred to as $100 \%$ (bottom panel). Data are shown as the mean $( \pm$ S.D.) of three independent experiments; ${ }^{*} p<0.05$. (C) A $\beta 40$ contents in the conditioned media of $\mathrm{Na}_{2} \mathrm{SeO}_{3}$ treated $\gamma$-30 cells (shaded bar) and untreated cells (solid bar) were determined by a colorimetric A 340 ELISA kit. Data are shown as the mean ( \pm S.D.) of triplicate measurements from a representative experiment and were analyzed by Student's $t$-test; $* p<0.05$. 
(A)

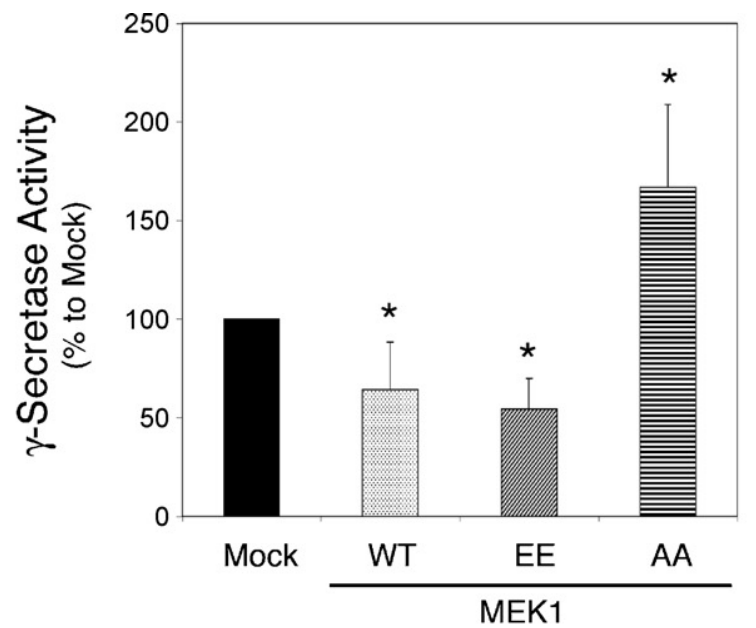

(B)

MEK1
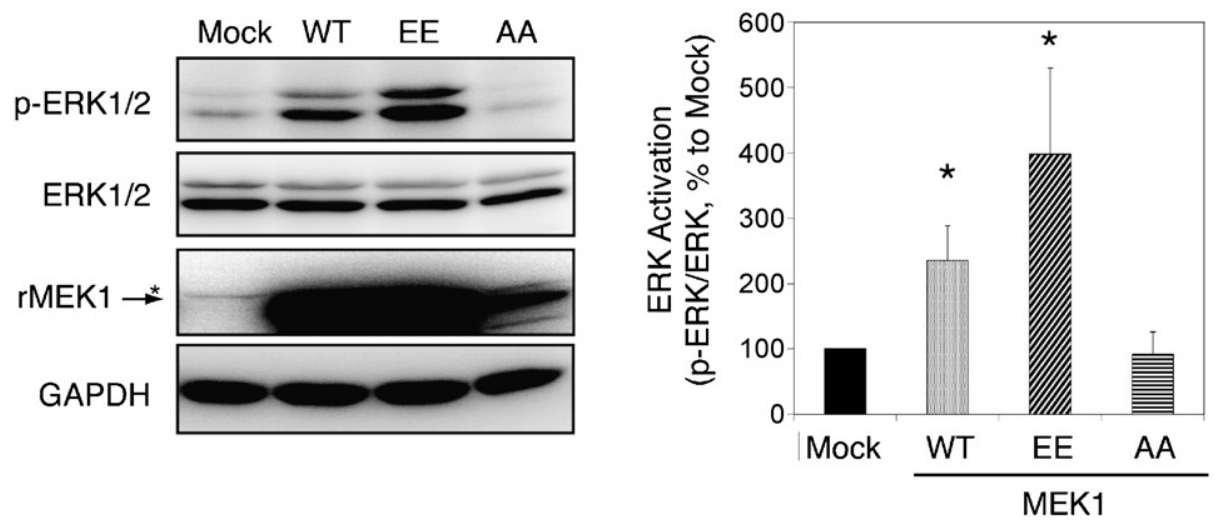

(C)

MEK1
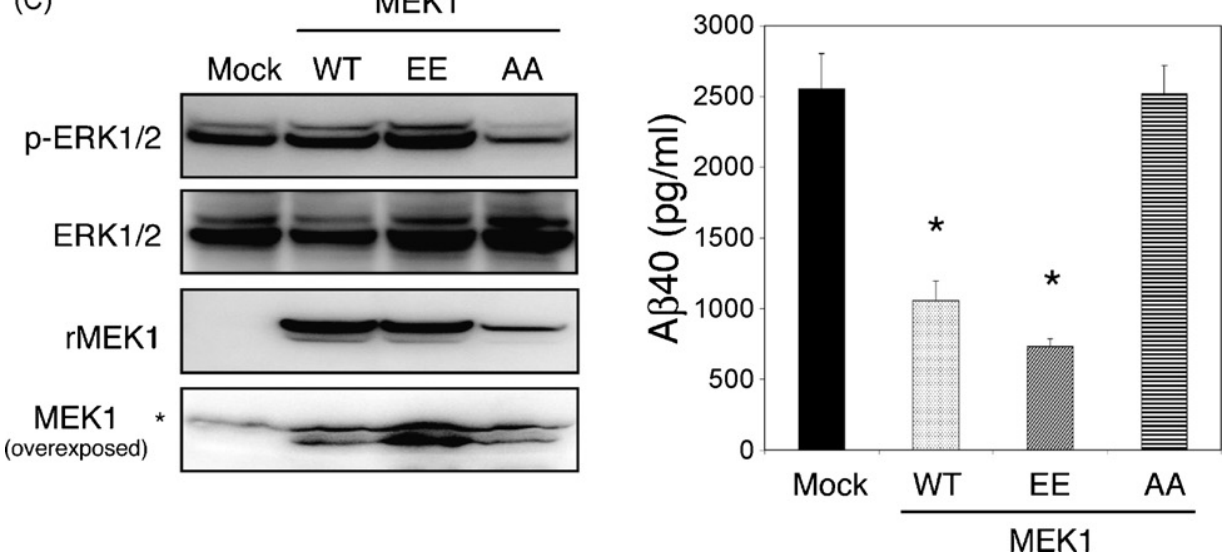

Fig. 2. Regulation of $\gamma$-secretase activity by MEK1, an immediate upstream activator of ERK1/2. (A) T20 cells were transfected with $0.1 \mu \mathrm{g} /$ well pRL-TK along with $0.5 \mu \mathrm{g} /$ well of an empty vector (Mock), the wild-type MEK1 (WT), a constitutively active MEK1 (MEK1-EE), or a dominant-negative MEK1 (MEK1-AA) in DMEM containing 10\% FBS for $48 \mathrm{~h}$ at $37^{\circ} \mathrm{C}$. Cells were harvested after briefly treated with EGF $(100 \mathrm{ng} / \mathrm{ml})$ for a duration of $3 \mathrm{~h}$. $\gamma$-Secretase activity of transfected cells was determined and normalized with the luminescence derived from the constitutively expressing Renilla luciferase, and the one of mock-transfected cells is referred to as $100 \%$ relative $\gamma$-secretase activity. Data are shown as the mean ( \pm S.D.) of triplicate measurements from three representative experiments; ${ }^{*} p<0.05$. (B) Prolonged activation of ERK1/2 induced by the MEK1-WT and the MEK1-EE mutant. Phosphorylated ERK1/2 (upper panel), total ERK1/2 (upper middle panel), MEK1 (lower middle panel), and GAPDH (bottom panel) in clarified lysates of transfected T20 cells were analyzed by Western blotting. The arrow indicates the expression of exogenous MEK1 (MEK1-WT, MEK1-EE, and MEK1-AA) that was not present in mock-transfected cells, while the asterisk denotes the endogenous MEK1. ERK activation induced by MEK1-WT and MEK1-EE is shown as the mean ( \pm S.D.) of three independent experiments; ${ }^{*} p<0.05$. (C) The attenuation of $A \beta$ production by the activation of ERK. A $\beta 40$ contents in the conditioned media of $\gamma$-30 cells that were transfected with an empty vector (Mock, solid bar), MEK1-WT (hatched bar), MEK1-EE (striped bar), or MEK1-AA (horizontal bar) were determined by an A 340 ELISA kit. Data are shown as the mean ( \pm S.D.) of triplicate measurements from a representative experiment. The phosphorylated ERK1/2 (top panel), total ERK1/2 (upper middle panel), and MEK1 (lower middle and bottom panels) were visualized to show the activation of ERK by MEK1-WT- and MEK1-EE-transfected cells. The expression of endogenous MEK1 was visualized by prolonged exposure of an immunoblot from one representative experiment (overexposed, bottom panel). 
activated either with sodium selenite, an inorganic salt known to activate ERK [6], or transfected with a constitutively active mutant of MEK1 (MEK1-EE), an immediate upstream activator of ERK $[8,16]$. Consistent with our hypothesis that $\gamma$-secretase activity should be suppressed due to persistent activation of the MEK-ERK pathway, sodium selenite-treated T20 cells exhibited significantly decreased $\gamma$-secretase activity, approximately a $50 \%$ reduction at $24 \mathrm{~h}$ of treat- ment (Fig. 1A). To better estimate the effect of ERK activation on $A \beta$ production, we resorted to a CHO-derived $\gamma$-30 cell line [9], which is a much superior cellular model to T20 cells in the determination of $A \beta$ production. Using $\gamma-30$ cells, we further verified that treatment with sodium selenite $(8 \mu \mathrm{M})$ induces prolonged stimulation of ERK over a 24-h span (Fig. 1B). Such prolonged activation of ERK resulted in marked reduction of secreted $A \beta 40$ in the con-
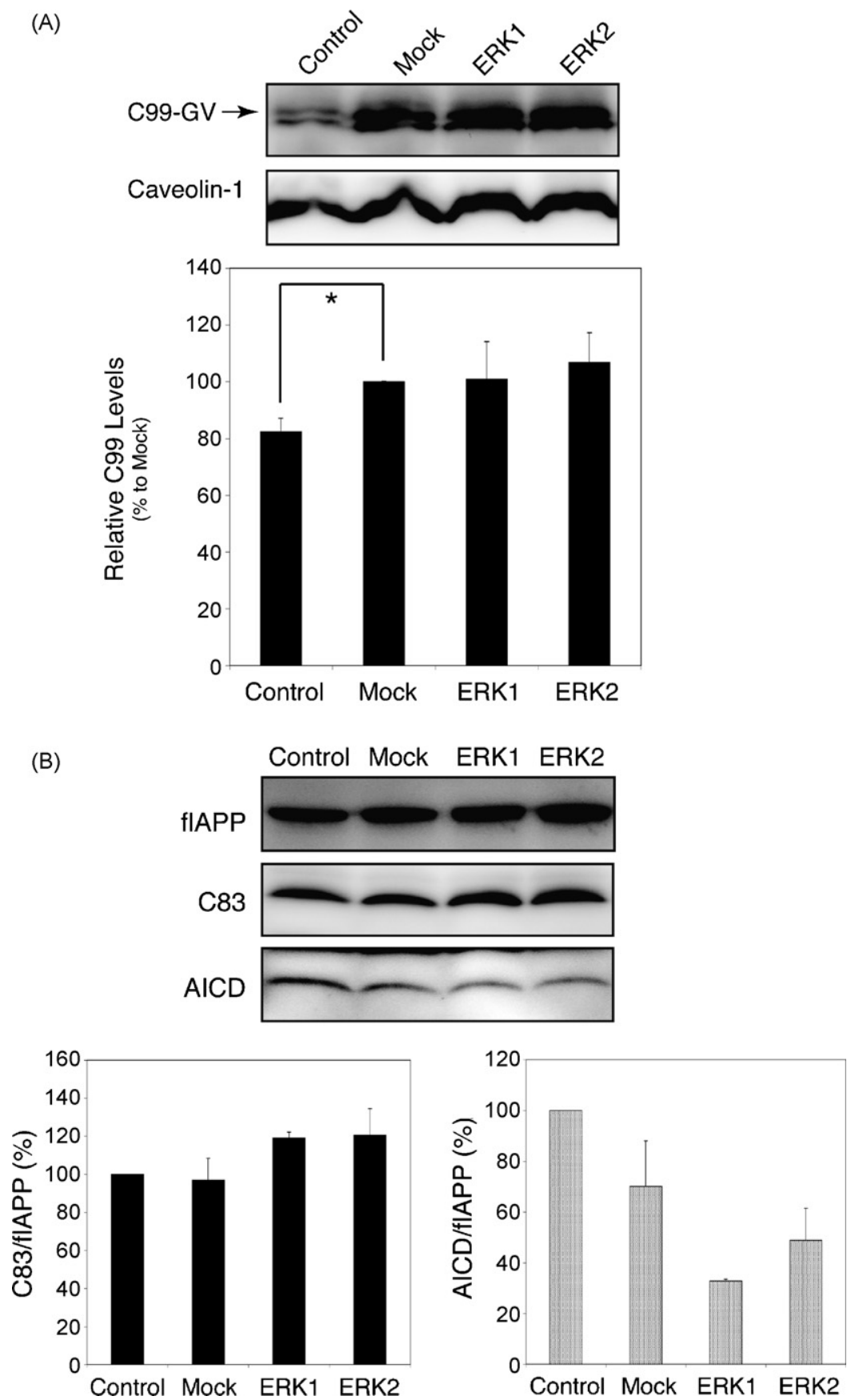

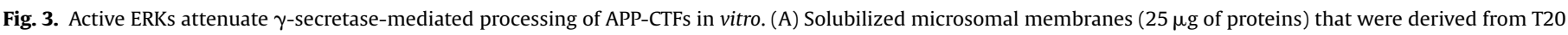

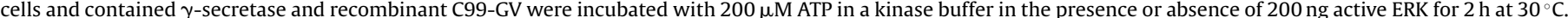

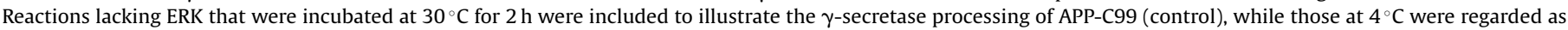

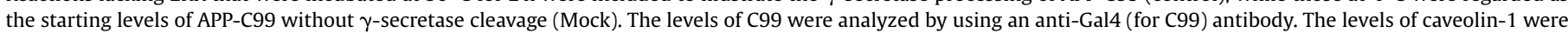

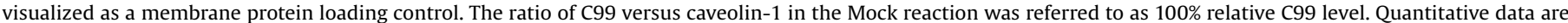

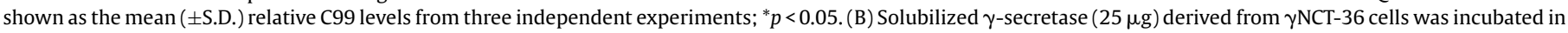

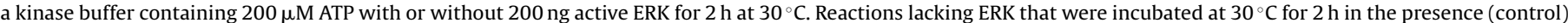

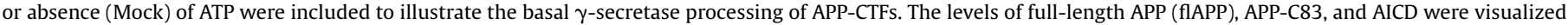

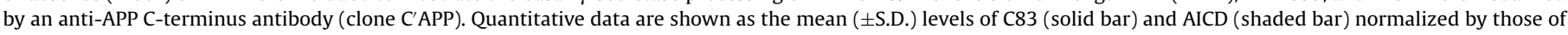
flAPP, a load control, from one representative experiment. 
ditioned media, i.e., an approximately 50\% decrease after $24 \mathrm{~h}$ of treatment (Fig. 1C). To substantiate the ERK-dependent suppression of $\gamma$-secretase activity, T20 cells were transfected with either wild-type MEK1 (WT) or MEK1-EE to specifically induce sustained activation of ERK. While T20 cells transfected with an empty vector (Mock) did not exhibit significant changes in $\gamma$-secretase activity, those transfected with MEK1-WT or MEK1-EE showed an approximately $50 \%$ reduction in $\gamma$-secretase activity, concomitant with the sustained activation of ERK (Fig. 2). In contrast, overexpression of the dominant-negative mutant of MEK1 (MEK1-AA) in T20 cells completely abolished ERK activation, resulting in a corresponding increase in $\gamma$-secretase activity (Fig. 2). Consistent with these data, the production of $A \beta$ in $\gamma-30$ cells transfected with either MEK-WT or MEK1-EE was significantly reduced, while that in those transfected with MEK1-AA was only marginally affected (Fig. 2C).

To further substantiate the functional role of MEK-ERK pathway in the regulation of $\gamma$-secretase activity, solubilized membrane preparation of T20 cells containing $\gamma$-secretase and the Gal4VP16tagged APP C-terminal fragment, C99-GV, was incubated with active purified ERK1 or ERK2. The control reactions lacking ERKs showed significantly reduced levels of C99-GV, indicative of the endogenous $\gamma$-secretase cleavage of C99-GV, while Mock reactions lacking ERKs at $4{ }^{\circ} \mathrm{C}$ showed the input levels of uncleaved C99-GV prior to being processed by $\gamma$-secretase (Fig. $3 \mathrm{~A}$ ). In the presence of either active ERK1 or ERK2, $\gamma$-secretase-mediated processing of C99-GV was completely blocked, evidenced by the accumulation of C99-GV to a comparable level as in Mock reactions (Fig. 3A). Furthermore, solubilized membrane preparation of $\gamma \mathrm{NCT}-36$ cells that overexpress APP and all four essential constituents of $\gamma$-secretase was incubated with or without active ERK1/2. We found that, in the presence of active ERKs, the accumulation of APP-C83 was increased in conjunction with a diminished production of AICD (Fig. 3B), suggesting the suppression of $\gamma$-secretase activity by ERKs.

To determine whether $\gamma$-secretase can be directly phosphorylated by ERK, solubilized $\gamma$-secretase isolated from the microsomal membranes of $\gamma$ NCT-36 cells was incubated with or without purified active ERKs in an in vitro kinase assay. We found that active ERKs can significantly induce the phosphorylation of NCT and fulllength PS1 (flPS1) in vitro, respectively (Fig. 4). These findings support a model in which ERKs directly interact with $\gamma$-secretase to attenuate its enzymatic activity through alterations in the phosphorylation of NCT and PS1. The present data thus strongly favor the idea that the ERK pathway plays an imperative role in maintaining the homeostasis of endogenous $\gamma$-secretase activity. Chemical reagents effective in promoting persistent ERK activation, such as sodium selenite, could provide appealing alternatives for the development of therapeutic strategies against the pathogenesis of AD.

The molecular mechanisms governing the physiological production of $A \beta$ in vivo remains mostly elusive. Only recently has it been possible to specifically dissect the $\gamma$-secretase-mediated cleavage of APP and to study the regulation of its proteolytic activity in a cellular context. Herein, we present novel findings demonstrating that activation of ERK MAPK by sodium selenite can effectively lower endogenous $\gamma$-secretase activity and $A \beta$ production through the phosphorylation of PS1 and NCT. Our present study delineates a molecular mechanism that may conform to an intracellular signaling network maintaining the homeostasis of $\gamma$-secretase activity and $A \beta$ production at a physiological level.

The beneficial effect of ERK activation by sodium selenite on suppressing $\gamma$-secretase is of particular interest. Currently, the association between brain selenium levels in $\mathrm{AD}$ patients is still unsettled [15], and its levels in cerebrospinal fluid do not differ significantly between AD patients and control groups [13].
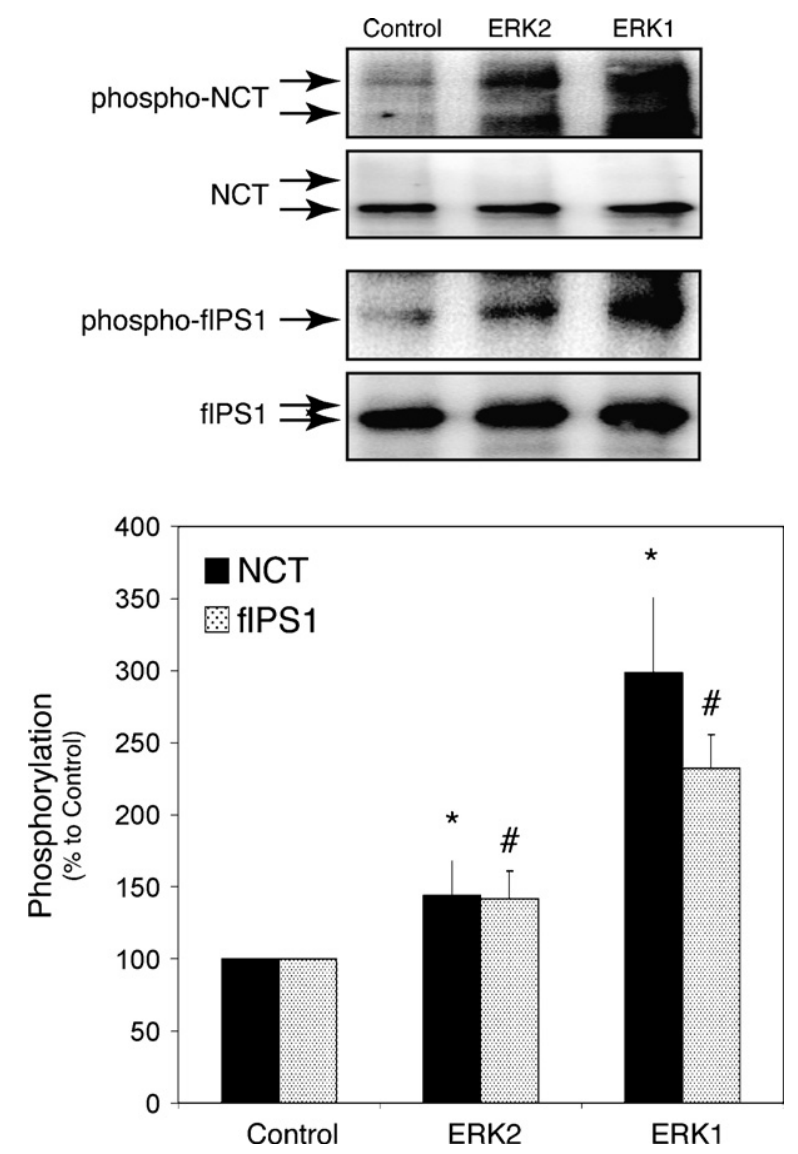

Fig. 4. ERK induces phosphorylation of NCT and PS1. $\gamma$-Secretase derived from $\gamma$ NCT-36 cells was partially purified by HIS-Select ${ }^{\mathrm{TM}}$ Cobalt Affinity Gel through affinity of the His-tagged NCT. Affinity-purified $\gamma$-secretase $(25 \mu \mathrm{g})$ was incubated with $200 \mathrm{ng}$ active ERK at $30^{\circ} \mathrm{C}$ for $1 \mathrm{~h}$. A reaction containing only affinity-purified $\gamma$-secretase was included as a control. Phosphorylated proteins were visualized by an anti-phospho-serine/threonine (phospho-NCT and phospho-flPS1) antibody. The relative phosphorylation levels (phosphorylated versus total protein) of flPS1 (shaded bar) and NCT (solid bar) were determined and compared with those of control that are referred to as $100 \%$ (histogram). Quantitative data are shown as the mean $( \pm$ S.D.) relative phosphorylation of flPS1 and NCT from at least three independent experiments. ${ }^{*} p<0.05$ versus control (NCT); ${ }^{*} p<0.05$ versus control (flPS1).

Nonetheless, the antioxidant potential of selenium agents has been shown to provide protection against diverse oxidative insults $[1,4]$. The administration of selenium has been shown to alleviate ROS-induced cell damage in animal models of various neurodegenerative diseases [15]. Our data thus shed light on the molecular mechanism underlying the neuroprotective effect of selenium that targets ERK MAPK and negatively regulates $\gamma$-secretase activity and $A \beta$ production. In addition, the present evidence also provides the proof-of-concept for identifying other selenium agents aimed at lowering $A \beta$ by using ERK as a pharmacological target. The protective role of ERK activation in $A D$ pathogenesis can be further elaborated by recent studies demonstrating that rasagiline-derived cholinesterase inhibitors promote ERK activation and stimulate $\alpha$-secretase activity [21,22]. Together, ERK activation could play a pivotal role in shifting APP processing toward the $\alpha$-secretaseinitiated non-amyloidogenic pathway and synergistically blocking $\gamma$-secretase-dependent $A \beta$ production.

Our new data strengthen the idea that the secretase-mediated proteolysis of the APP is subject to multiple levels of regulation by intracellular pathways. Thus, our current and previous findings that JNK and ERK pathways modulate $\gamma$-secretase activity are in accordance with the notion that both oxidative stress (JNK activation) 
and abnormalities in MAPK signaling (ERK inhibition) are necessary for the propagation of $\mathrm{AD}[11,23]$. Thus, intrinsic signaling cascades may converge on $\gamma$-secretase and constitute such a delicate regulatory system that governs the homeostasis of $A \beta$ production via the counteracting effects of JNK and ERK.

\section{Acknowledgments}

We thank Dr. Michael Wolfe for providing compound E, DAPT, and the $\gamma-30$ cell line. We are also grateful to Drs. Jen-Leih $\mathrm{Wu}$, Nin-Nin Chuang, and John Yu for generous supports. The authors thank the Core Facility of the Institute of Cellular and Organismic Biology, Academia Sinica, for technical support. This study was supported by the National Science Council, Taiwan (NSC 932320-B-001-037 and 94-2320-B-001-004), the National Research Program for Genomic Medicine, Taiwan (NSC 94-3112-B-001-001 and 95-3112-B-001-006), and Academia Sinica (to Y.-F.L.).

\section{References}

[1] J. Chen, M.J. Berry, Selenium and selenoproteins in the brain and brain diseases, J. Neurochem. 86 (2003) 1-12.

[2] W.P. Esler, M.S. Wolfe, A portrait of Alzheimer secretases-new features and familiar faces, Science 293 (2001) 1449-1454.

[3] D. Gianni, N. Zambrano, M. Bimonte, G. Minopoli, L. Mercken, F. Talamo, A. Scaloni, T. Russo, Platelet-derived growth factor induces the beta-gammasecretase-mediated cleavage of Alzheimer's amyloid precursor protein through a Src-Rac-dependent pathway, J. Biol. Chem. 278 (2003) 9290-9297.

[4] G.I. Giles, F.H. Fry, K.M. Tasker, A.L. Holme, C. Peers, K.N. Green, L.O. Klotz, H. Sies, C. Jacob, Evaluation of sulfur, selenium and tellurium catalysts with antioxidant potential, Org. Biomol. Chem. 1 (2003) 4317-4322.

[5] J. Hardy, Amyloid, the presenilins and Alzheimer's disease, Trends Neurosci. 20 (1997) 154-159.

[6] H. Hu, C. Jiang, G. Li, J. Lu, PKB/AKT and ERK regulation of caspase-mediated apoptosis by methylseleninic acid in LNCaP prostate cancer cells, Carcinogenesis 26 (2005) 1374-1381.

[7] T. Iwatsubo, The gamma-secretase complex: machinery for intramembrane proteolysis, Curr. Opin. Neurobiol. 14 (2004) 379-383.

[8] H. Jaaro, H. Rubinfeld, T. Hanoch, R. Seger, Nuclear translocation of mitogenactivated protein kinase kinase (MEK1) in response to mitogenic stimulation, Proc. Natl. Acad. Sci. U.S.A. 94 (1997) 3742-3747.

[9] W.T. Kimberly, M.J. LaVoie, B.L. Ostaszewski, W. Ye, M.S. Wolfe, D.J. Selkoe, Gamma-secretase is a membrane protein complex comprised of presenilin nicastrin, Aph-1, and Pen-2, Proc. Natl. Acad. Sci. U.S.A. 100 (2003) 6382-6387.
[10] G.X. Li, H. Hu, C. Jiang, T. Schuster, J. Lu, Differential involvement of reactive oxygen species in apoptosis induced by two classes of selenium compounds in human prostate cancer cells, Int. J. Cancer 120 (2007) 2034-2043.

[11] Y.F. Liao, B.J. Wang, H.T. Cheng, L.H. Kuo, M.S. Wolfe, Tumor necrosis factor-alpha, interleukin-1beta, and interferon-gamma stimulate gammasecretase-mediated cleavage of amyloid precursor protein through a JNK-dependent MAPK pathway, J. Biol. Chem. 279 (2004) 4952349532.

[12] M. Mei, B. Su, K. Harrison, M. Chao, S.L. Siedlak, L.A. Previll, L. Jackson, D.X. Cai, X. Zhu, Distribution, levels and phosphorylation of Raf-1 in Alzheimer's disease, J. Neurochem. 99 (2006) 1377-1388.

[13] I. Meseguer, J.A. Molina, F.J. Jimenez-Jimenez, M.V. Aguilar, C.J. Mateos-Vega, M.J. Gonzalez-Munoz, F. de Bustos, M. Orti-Pareja, M. Zurdo, A. Berbel, E. Barrios, M.C. Martinez-Para, Cerebrospinal fluid levels of selenium in patients with Alzheimer's disease, J. Neural Transm. 106 (1999) 309-315.

[14] E. Rudolf, K. Rudolf, M. Cervinka, Selenium activates p53 and p38 pathways and induces caspase-independent cell death in cervical cancer cells, Cell Biol. Toxicol. 24 (2008) 123-141.

[15] U. Schweizer, L. Schomburg, N.E. Savaskan, The neurobiology of selenium: lessons from transgenic mice, J. Nutr. 134 (2004) 707-710.

[16] R. Seger, D. Seger, A.A. Reszka, E.S. Munar, H. Eldar-Finkelman, G. Dobrowolska, A.M. Jensen, J.S. Campbell, E.H. Fischer, E.G. Krebs, Overexpression of mitogenactivated protein kinase kinase (MAPKK) and its mutants in NIH 3T3 cells. Evidence that MAPKK involvement in cellular proliferation is regulated by phosphorylation of serine residues in its kinase subdomains VII and VIII, J. Biol. Chem. 269 (1994) 25699-25709.

[17] D.J. Selkoe, Alzheimer's disease: genes, proteins, and therapy, Physiol. Rev. 81 (2001) 741-766.

[18] R.E. Tanzi, L. Bertram, Twenty years of the Alzheimer's disease amyloid hypothesis: a genetic perspective, Cell 120 (2005) 545-555.

[19] Q. Wang, Q.G. Zhang, D.N. Wu, X.H. Yin, G.Y. Zhang, Neuroprotection of selenite against ischemic brain injury through negatively regulating early activation of ASK1/JNK cascade via activation of PI3K/AKT pathway, Acta. Pharmacol. Sinica 28 (2007) 19-27.

[20] M.S. Wolfe, The secretases of Alzheimer's disease, Curr. Top Dev. Biol. 54 (2003) 233-261.

[21] M. Yogev-Falach, T. Amit, O. Bar-Am, M. Weinstock, M.B. Youdim, Involvement of MAP kinase in the regulation of amyloid precursor protein processing by novel cholinesterase inhibitors derived from rasagiline, FASEB J. 16 (2002) 1674-1676.

[22] M.B. Youdim, O. Bar Am, M. Yogev-Falach, O. Weinreb, W. Maruyama, M. Naoi, T. Amit, Rasagiline: neurodegeneration, neuroprotection, and mitochondrial permeability transition, J. Neurosci. Res. 79 (2005) 172-179.

[23] X.Zhu, R.J. Castellani, A. Takeda, A. Nunomura, C.S. Atwood, G. Perry, M.A. Smith, Differential activation of neuronal ERK, JNK/SAPK and p38 in Alzheimer disease: the 'two hit' hypothesis, Mech. Ageing Dev. 123 (2001) 39-46.

[24] X. Zhu, A.K. Raina, G. Perry, M.A. Smith, Alzheimer's disease: the two-hit hypothesis, Lancet Neurol. 3 (2004) 219-226.

[25] X.Zhu, Z.Sun, H.G. Lee, S.L. Siedlak, G. Perry, M.A. Smith, Distribution, levels, and activation of MEK1 in Alzheimer's disease, J. Neurochem. 86 (2003) 136-142. 